\title{
STUDY OF THE FLUX DENSITY VARIATION IN AND AROUND THE PLANET JUPITER USING 16" LX200GPS SCHMIDT- CASSEGRAIN TELESCOPE AT NATIONAL OBSERVATORY, NAGARKOT, NEPAL
}

\author{
N. R. Ghimire*, S. R. Shahi** and B.Aryal* \\ *Central department of Physics, Tribhuvan University, Kirtipur, Nepal. \\ **B.P. Koirala Memorial Planetarium, Observatory and Science Museum Development Board, Kathmandu, Nepal.
}

\begin{abstract}
We present a study of relative flux density in and around the planet Jupiter using images taken from National Observatory located at Nagarkot, Nepal during the period from November, 2008 and January, 2010. For this Meade 16 inch LX200GPS Schmidt-Cassegrain telescope is used. We processed the image in the software ALADIN2.5. The values of relative flux density of 2,50,000 pixels are used for the study. At first, the observed two-dimensional image of the planet is divided into four quadrants. Each quadrant is subdivided into three sections. We studied the variation of relative flux density along 12 directions. The relative flux density per pixel is found to lie in the range 138.17 to 157.36 . The maximum value of relative flux density per pixel is found along the eastern direction and the minimum value is found along the northern direction. Thus the absorption feature is found to be prominent along the northern direction. The emission of less amount of flux from the second quadrant is due to the absorption by the materials in this section. In addition, the role of the inhomogeneous absorption by the on-going gravitational contraction in the planet cannot be identified. Due to the inhomogeneous absorption feature, we studied the outer contours of all sections. The values of total amount of flux per pixel in the outer layers of the first, second, third and fourth quadrants are found to be $81.79,82.37,82.44$ and 82.49 respectively. Surprisingly, this value is found to be least in the first quadrant. This suggests that the outer part is found to be selective whereas the inner part is emissive.
\end{abstract}

Key-words: Observation; Telescope; Jupiter; Flux density.

\section{INTRODUCTION}

The planet Jupiter has a unique position in the Solar system. As one of the brightest objects in the sky, having visual magnitude of 2.6 and angular diameter up to 44 arc second at opposition, it was named after the most powerful god of the mythology. It is a fascinating object for all observers, professional and amateurs, who can easily detect the main features of its atmosphere and watch the Galilean satellites. Apart from its attractive aspect, Jupiter is an object of special interest for planetologists. At 5.2 AU (Astronomical Unit, 1 $\mathrm{AU}=149,597,870.66 \mathrm{~km}$ ) from the Sun, it is the closest and the most massive of the giant planets, which extend in the outer solar system up to heliocentric distances of $30 \mathrm{AU}^{1}$. Its mass accounts to 318 terrestrial masses, and its diameter is 11 times the terrestrial one. As all giant planets, Jupiter has a low density $\left(1.31 \mathrm{~g} / \mathrm{cm}^{3}\right)$ which reects its chemical composition, mostly dominated by hydrogen and helium. Jupiter has a fast rotation around its polar axis (9 hours 55 minutes) which induces a characteristic structure of belts and zones, parallel to the equator. As the other giant planets, it has a ring system and many satellites; its ring is very tenuous ${ }^{2}$. The planet is surrounded by 63 satellites known by the beginning of the year $2006^{1}$, including the four main ones discovered by Galileo. Another characteristic of Jupiter is its magnetosphere, more extended than the ones of the other giant planets, and comparable in some regards to the terrestrial magnetosphere ${ }^{3}$.

In the present work, we have planned to observe and study the planet Jupiter using 16 inch LX200GPS SchmidtCassegrain telescope located at the National Observatory, Nagarkot (located $32 \mathrm{~km}$ east of Kathmandu). The SchmidtCassegrain is a catadioptric telescope that combines a cassegrain rector's optical path with a Schmidt corrector plate to make a compact astronomical instrument that uses simple spherical surfaces ${ }^{4}$. This is an optical telescope. Optical astronomy refers to the observation and study of light. It includes the non-visible and visible light in the electromagnetic spectrum. In all forms of observational astronomy, we collect information about the heavenly bodies using telescopes and analyze this information to come to valuable predictions and conclusions. In optical astronomy, we take into account visible light while in other forms, we use other wavelengths of the electromagnetic spectrum. Optical images are drawn by hand, with information from photographic devices and later on using digital detectors,

Author for Correspondence: B. Aryal, Central department of Physics, Tribhuvan University, Kirtipur, Nepal. 
particularly CCD (charge coupled devices).

We intend to study the flux density variation in the outer layer of the planet Jupiter. The prime objectives of this work are as follows: (1) We intend to operate and calibrate the telescope named 1600 LX200GPS Schmidt-Cassegrain” installed in the National Observatory located at Nagarkot. (2) We intend to carry out a few observational runs in the period 2008-2010 focusing the planets and their moons. (3) We focus our attention to the planet Jupiter. Our interest is to capture Jupiter's images and process them in the data reduction software, particularly for relative flux density. (4) Our motive is to model Jupiter's outer environment from the study of variation of relative flux density. (6) Finally, we intend to discuss the cause of selective absorption in the outer layer of the planet Jupiter.

\section{OBSERVATION}

Nagarkot is situated at latitude $27^{\circ} 41^{\prime} 06^{\prime \prime} \mathrm{N}$ and longitude 85'31'00" E, 32 km east of the capital city, Kathmandu (Fig. 1a). High altitude, clear sky, narrow population and noiseless environment are the basic features of Nagarkot that provided the idea of constructing the observatory there. Because of its high altitude, the weather is cold throughout the year. Most of the time the climatic condition is a bit humid, therefore, to minimize errors owing to humidity, we used an electric absorbent inside the dome while taking observations. The National Observatory at Nagarkot is the only observatory in our nation and is also popular as Nagarkot observatory. It constitutes a two-storey building in which a 1600 LX200GPS Schmidt-Cassegrain telescope has been installed (Fig. 1b). In addition there is a rest house that offers all the facilities to the observers.
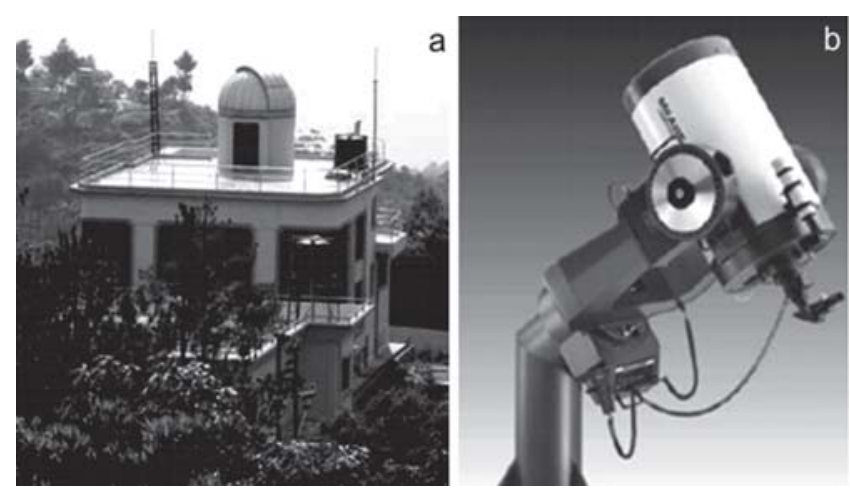

Figure 1: (a) A scenic view of National Observatory at Nagarkot. (b) Meade 1600 LX200GPS Schmidt-Cassegrain telescope.

We visited the observatory in four sessions. The very first session was in November, 2008. It was winter, the sky was clear and we tried to take observations under slightly humid conditions. Since it was our first visit, we spent the entire night trying to learn the techniques to handle the telescope and to produce images. Unfortunately, the images that we obtained were not satisfactory; they were absurd. The second time that we went there was in March, 2009. The winter was on its brink but the sky was not clear the entire night. However, we tried to take images whenever possible. By that time we had learned to handle the telescope properly. The image of the planet Jupiter that we worked on belongs to this session. The major achievement that we made was in the third session, during October, 2009. We were able to produce focused images. However, we had one more session in January, 2010. In that session, we produced images of not only Jupiter, but also of Saturn, moon (of Earth) and the star Polaris (Fig. 2). By the end of that session, we had been able to handle the telescope with ease and to produce good images, good enough to work on.

To help us identify the objects in the sky, we used the software CYBERSKY3.0 $0^{5}$. It is an accurate planetarium program that provides an excellent way to learn about astronomy and explore the sky visible in the distant past, the present, and the future. The program makes it easy to identify the objects we see in the sky and find the objects we want to see. Before observing any object, we must define a reference frame with the telescope at its center. For this we took into account two prominent stars - Rigel and Betelgeuse. In the select menu of the telescope, we chose the two-star alignment. We manually set the telescope so that the Rigel was at the center of the field of view. After this star was set in the memory of the telescope, we set it again to focus on the Betelgeuse. When this star was set in the memory, we got the message “Alignment Successful”. This message ensured that the reference frame was set and we were ready to use the telescope to view any possible object in the sky. Once a reference frame is set, the telescope works automatically as per the commands given to it through the AUTOSTAR II handbox. For example, when we give the command to go to the planet Jupiter, the telescope would rotate itself to focus on the Jupiter. The planet would appear in or near the field of view, depending on the accuracy with which we fix a reference frame. Once the telescope aligns itself to focus on the desired object, the only effort we have to make is to bring it in the center of the field of view. Once the desired object appears in the center of field of view of the telescope, the eyepiece is replaced by a webcam connected to the computer. The software that we used to obtain the images on the computer is AUTOSTAR SUITE3.085. The installation CD came along with the telescope and is a copyright of Meade Instruments Corporation, USA ${ }^{5}$.
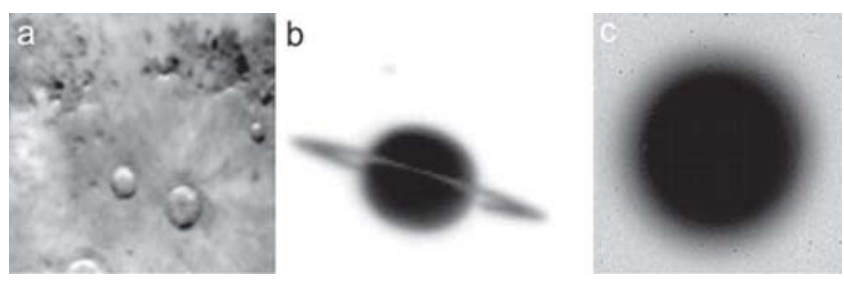

Figure 2: FITS images of moon's surface (a) Saturn (b) and Jupiter (c). There are $500 \times 500$ pixels in the field of view. Therefore the information regarding relative flux density is known for 2,50,000 pixels. We processed the images in the software ALADIN2.5.

There are two simultaneous processes that should be followed to remove the unwanted noise from the image obtained. The first one is to obtain a dark frame and subtract it from the raw light image. The other one is to obtain a at field 
image and use it to correct for variations in pixel response uniformity across the area of our dark-subtracted image.

\section{IMAGE PROCESSING}

The image captured by the telescope is a two-dimensional projection of the actual, three dimensional structure of the Jupiter (see Fig. 2c). However, to proceed further, we need to locate its center. There is an option in the image-processing part of AUTOSTAR SUITE that enables us to locate the center approximately. We took 12 measures of those approximate values and averaged them. The center was found at the pixel with coordinates $(x, y)=(312.40,211.92)$

The further processing of the FITS image was done in ALADIN2.5. ALADIN, an interactive sky atlas developed at Centre de Donnees astronomiques de Strasbourg (CDS) in France, is a service providing simultaneous access to digitized images of the sky, astronomical catalogues, and databases. The driving motivation is to facilitate direct, visual comparison of observational data at any wavelength with images of the optical sky, and with reference catalogues. On our FITS image, we created contours of iso-potential (in our case relative flux density) surfaces. We observed that the inner contours show mild fluctuations while the outer ones show severe fluctuations (see Fig. 3a), that is, the contour levels on the surface are almost homogeneous while those in the atmosphere are not. We decided to study the variation of relative flux density along different directions and in different regions. We drew 12 lines that meet at the center such that the angle between any two adjacent lines is almost $30^{\circ}$ (see Fig. 3a). We clicked on each pixel from the inner to the outer region that the lines pass through. We noted the position and relative flux density associated with each of the clicked pixels. Similarly, since the outermost two contours show most severe fluctuations, we divided the region between these contours into 12 regions, separated by the 12 lines (Fig. 3a). We clicked on every pixel lying within these regions and noted the position and relative flux density as we did for the 12 lines.

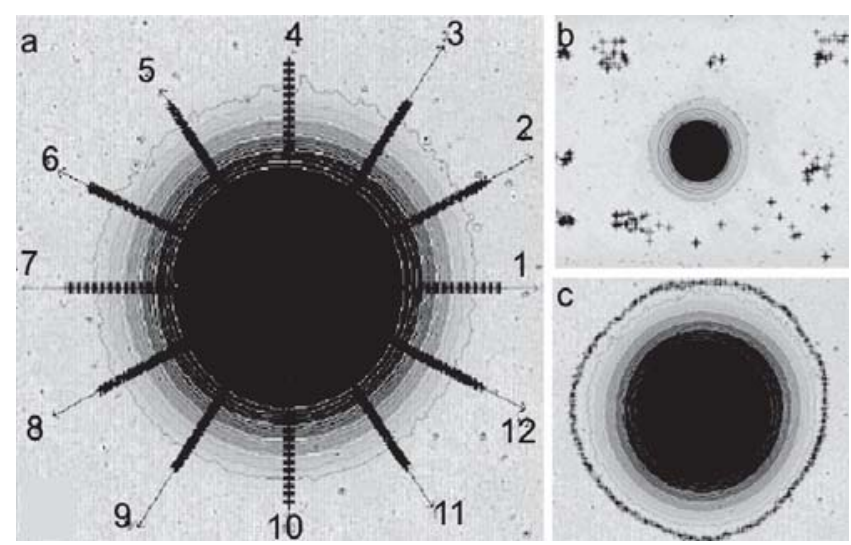

Figure 3: Image of Jupiter showing contours, axes and ticks in the image processing software ALADIN 2.5: The image (a) shows 12 axes meeting at the center (b) shows how we obtained background while the lower one (c) shows how we obtained homogeneous background.

\section{RESULTS}

In this section we give the results concerning the variation of relative flux density in and around the planet Jupiter. For this we used the image captured by us using Meade 16-inch LX200GPS Schmidt-Cassegrain telescope at Nagarkot Observatory during March, 2009. Our prime interest is to study the variation of relative flux density around the Jupiter's surface. The contours on the image of the Jupiter are almost homogeneous in the inner region. This implies homogeneous absorption and reaction of flux density in the central region. As we move from inner to outer contours, we can see increase in fluctuations. The outermost contour shows maximum fluctuations which reveal the features of the atmosphere (see Fig. 3a), i.e., the absorption and reaction features are inhomogeneous in the atmosphere.

We are going to discuss the variation of relative flux density along the 12 lines from the inner to the outer region (see Fig. 4). The values of the relative flux density for these lines have been obtained from ALADIN2.5 while the associated graphs have been derived from ORIGIN5.0.

Now we are going to discuss the values of relative flux density for line 1 which lies towards east in the first quadrant (i.e., at an angle of $0^{\circ}$ with the horizontal axis). The values are listed on the table 1 . We took the first column of Table 1 to ORIGIN5.0 and labeled it with $r$. We calculated the standard error for the column of $F_{v}$ and plotted the graph of $\ln \left(F_{v}\right)$ against $r$, as shown in Fig. 4. At first, the slope of the graph is almost constant and tends to zero. As the value of $r$ increases, the value of relative flux density $F_{v}$ goes on decreasing so that the slope goes on changing. The second order polynomial fit of the data is in close agreement with the linear fit. The polynomial fit is shown by a solid line while the linear fit by a dashed line. There is not much deviation of the data from the fitted curves in the inner region but we can see prominent deviation on both sides of the fitted curves as we go to the outermost region. In this graph and all the others, a solid circle represents a value of $\ln \left(F_{v}\right)$ corresponding to a value of $r$. Each of these circles is attached to two bars, one on its upper side and the other on its lower side. These bars are called error bars. The error is actually the standard error for the values of relative flux density as obtained through statistical analysis. With regards to line 1 , the value of the slope for the linear fit is -0.029 and that of the intercept is 5.81 . The value of relative flux density per pixel is maximum for line 1.

Variation of relative flux density along line 2: As in graph for line 1 , the slope at first tends to zero and is constant. As the value of $\mathrm{r}$ increases, the slope goes on changing. The distribution of relative flux density is uniform in the beginning and as we move to the outer region, we can see fluctuations in these values. The values of the slope and intercept for the linear fit are -0.289 and 5.72 respectively. These values do not differ so much from those for line 1. Similarly, the linear and polynomial fit in this case is also in close agreement. 
Table 1: Values of relative flux density along line 1: The first column gives the serial number for the clicks (i.e, pixels) showing that the clicks are from inner to outer region, the second and third columns give the pixel coordinates ( $\mathrm{x}$ and $\mathrm{y}$ ), and the last column gives the value of relative flux density $\left(F_{v}\right)$ for each pixel. The next eight columns repeat the first four.

\begin{tabular}{|c|c|c|c|c|c|c|c|c|c|c|c|}
\hline $\mathrm{P}$ & $\boldsymbol{x}$ & $\boldsymbol{y}$ & $\boldsymbol{F}_{\boldsymbol{v}}$ & $\mathrm{P}$ & $\boldsymbol{x}$ & $\boldsymbol{y}$ & $\boldsymbol{F}_{\boldsymbol{v}}$ & $\mathrm{P}$ & $\boldsymbol{x}$ & $\boldsymbol{y}$ & $\boldsymbol{F}_{\boldsymbol{v}}$ \\
\hline 1 & 354.31 & 268.00 & 254.73 & 21 & 373.06 & 267.94 & 215.82 & 41 & 392.44 & 268.00 & 79.25 \\
\hline 2 & 355.25 & 268.00 & 255.09 & 22 & 373.75 & 267.94 & 215.82 & 42 & 393.50 & 267.94 & 102.29 \\
\hline 3 & 356.19 & 267.94 & 254.33 & 23 & 374.50 & 268.00 & 198.81 & 43 & 394.50 & 268.00 & 87.87 \\
\hline 4 & 356.94 & 267.94 & 254.33 & 24 & 375.56 & 267.94 & 190.57 & 44 & 395.56 & 268.00 & 88.03 \\
\hline 5 & 357.81 & 267.94 & 254.94 & 25 & 376.50 & 268.00 & 174.19 & 45 & 396.56 & 268.00 & 77.32 \\
\hline 6 & 358.63 & 268.00 & 252.71 & 26 & 377.50 & 268.00 & 181.71 & 46 & 397.50 & 268.00 & 84.77 \\
\hline 7 & 359.63 & 268.00 & 254.62 & 27 & 378.56 & 268.00 & 161.36 & 47 & 398.31 & 268.00 & 73.22 \\
\hline 8 & 360.63 & 268.00 & 250.26 & 28 & 379.44 & 267.94 & 175.36 & 48 & 399.44 & 268.00 & 89.74 \\
\hline 9 & 361.69 & 268.00 & 251.51 & 29 & 380.50 & 268.00 & 157.49 & 49 & 400.56 & 268.00 & 60.23 \\
\hline 10 & 362.63 & 268.00 & 250.37 & 30 & 381.38 & 267.94 & 156.14 & 50 & 401.56 & 268.00 & 81.76 \\
\hline 11 & 363.50 & 268.00 & 251.73 & 31 & 382.38 & 268.00 & 154.31 & 51 & 402.50 & 268.00 & 62.16 \\
\hline 12 & 364.44 & 268.00 & 246.90 & 32 & 383.56 & 267.94 & 146.52 & 52 & 403.50 & 267.94 & 79.05 \\
\hline 13 & 365.44 & 268.00 & 246.98 & 33 & 384.63 & 267.94 & 124.65 & 53 & 404.50 & 268.00 & 60.99 \\
\hline 14 & 366.56 & 267.94 & 232.02 & 34 & 385.50 & 268.00 & 135.78 & 54 & 405.44 & 268.00 & 71.87 \\
\hline 15 & 367.38 & 268.00 & 245.67 & 35 & 386.38 & 267.94 & 114.37 & 55 & 406.44 & 268.00 & 65.57 \\
\hline 16 & 368.25 & 268.00 & 232.51 & 36 & 387.44 & 267.94 & 119.21 & 56 & 407.38 & 268.00 & 85.66 \\
\hline 17 & 369.38 & 267.94 & 241.72 & 37 & 388.56 & 268.00 & 102.39 & 57 & 408.50 & 268.00 & 56.31 \\
\hline 18 & 370.38 & 267.94 & 214.09 & 38 & 389.69 & 267.94 & 117.20 & 58 & 409.50 & 267.94 & 84.14 \\
\hline 19 & 371.38 & 267.94 & 226.27 & 39 & 390.50 & 268.00 & 85.70 & 59 & 410.44 & 268.06 & 49.07 \\
\hline 20 & 372.38 & 267.94 & 216.17 & 40 & 391.50 & 268.00 & 109.37 & 60 & 411.56 & 268.06 & 78.72 \\
\hline
\end{tabular}

Variation of relative flux density along line 3 : This graph differs slightly from the plots for line 1 and line 2 . The other two plots are almost steady in the beginning while this plot shows a slight inhomogeniety in the region around $r=25$. The slope is almost constant in the beginning and goes on changing as the value of $r$ increases. The fluctuations are almost steady in the inner region but severe in the outer region of the planet. The linear and polynomial fits differ only slightly as for line 1 and line 2 . The values of slope and intercept for the linear fit are -0.024 and 5.71 respectively.

Variation of relative flux density along line 4: As compared to line 3 , this graph is more homogeneous in its appearance. The fluctuations are less severe in the inner and outer regions. The other features are similar to the earlier three lines. The linear and polynomial fits do not differ significantly. The values of slope and intercept for the linear fit are -0.031 and 5.69 respectively. The value of relative flux density per pixel is least for line 4 .

Variation of relative flux density along line 5: Now we are in the second quadrant and the graph reveals the similar features as the other four graphs we discussed earlier. In the inner region of the planet, the distribution of relative flux density is almost homogeneous as in the case of line 4 but in the outer region, the fluctuations are more severe than in the case of line 4 . The linear and polynomial fits are in closer agreement than line 4 . The values of the slope and intercept for linear fit are -0.024 and 5.73 respectively.

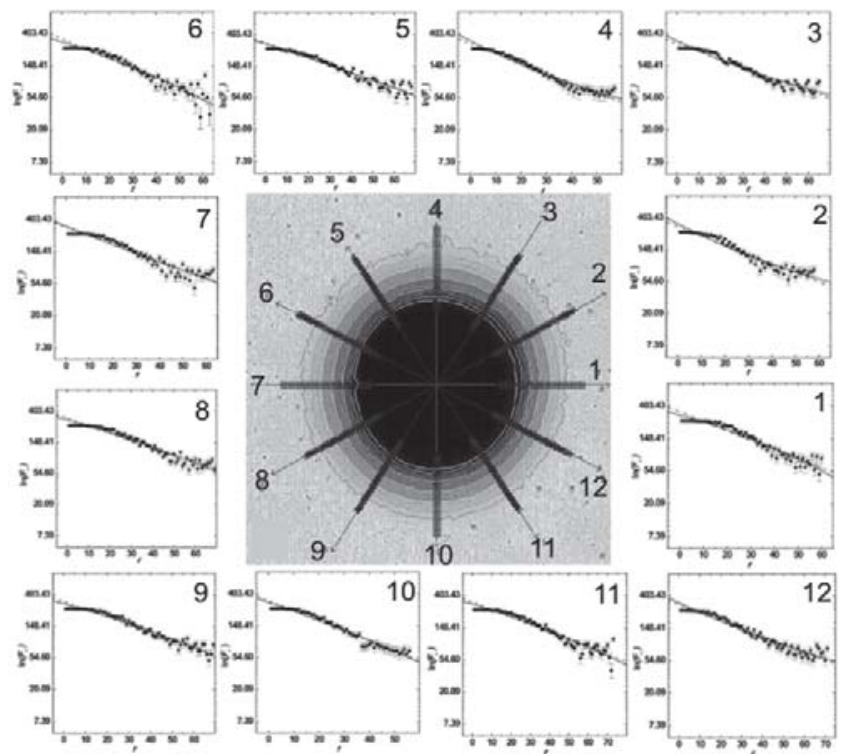

Figure 4: Variation of relative flux density along line 1-12: Here $\mathrm{F}_{v}$ represents the value of relative flux density obtained from Table 1 and therefore, $\ln \left(\mathrm{F}_{v}\right)$ represents the natural logarithm. Similarly, $r$ represents serial number for the clicks; the increasing value of $r$ shows that the clicks are from inner to outer region of the image of planet Jupiter. The dashed and solid lines represent linear and second order polynomial fits respectively. The solid circles with error bars are the observed values of relative flux density. The standard error of the values of relative flux density is taken as the error in the error bars.

Variation of relative flux density along line 6: As earlier, the slope is almost zero and constant in the beginning. As we 
move towards the outer region, the value of $\ln \left(F_{v}\right)$ goes on decreasing so that the slope changes. In the inner region of the planet, the distribution of relative flux density is homogeneous as in the case of line 5 but in the outer region, the fluctuations are more severe. The linear and polynomial fits are in less closer agreement than in the case of line 5 . The values of slope and intercept for the linear fit are -0.030 and 5.82 respectively.

Variation of relative flux density along line 7: The slope is almost constant in the beginning as for the earlier lines. This graph does not appear much different from that for line 6 . The fluctuations are steady in the inner region just like line 6 but in the outer region, the fluctuations are less severe than those for line 6 . The linear and polynomial fits are in closest agreement as compared to all the other earlier lines. The slope and intercept for the linear fit is -0.027 and 5.76 respectively.

Variation of relative flux density along line 8: This graph is more homogeneous in appearance than the earlier lines. As compared to line 7 , the fluctuations in the inner region are steadier and those in the outer region are less severe. The linear and polynomial fits are in close agreement but in less closer agreement as compared to line 7 . The values of slope and intercept for the linear fit are -0.024 and 5.77 respectively.

Variation of relative flux density along line 9: Just like the graph for line 8, this graph is homogeneous in appearance. The slope is constant initially and goes on changing as the value of $r$ increases. The rate of fluctuations increases from the inner to the outer region of the planet. The fluctuations in the inner and outer regions are almost similar to those for line 8. The slope and intercept for the linear fit are -0.024 and 5.76 respectively. The linear and polynomial fits are in good agreement as earlier.

Variation of relative flux density along line 10: Initially the graph is steady with slope almost constant. While moving to the outer region the slope changes. There is slight inhomogeneity in the fluctuations around $r=35$. Beyond that the fluctuations are almost as severe as for line 9 . There is a good agreement between linear and polynomial fits as before. The slope and intercept for the linear fit are -0.030 and 5.77 .

Variation of relative flux density along line 11: As compared to line 10 , the graph is homogeneous in the inner region. But beyond $r=45$, there are more severe fluctuations above and below the best fitted curves. The best fitted linear and polynomial curves are in good agreement. The slope and intercept for the linear fit are -0.023 and 5.78 respectively.

Variation of relative flux density along line 12: The slope is almost zero and constant in the beginning. As we move towards the outer region, the homogeneity in the curve breaks earlier than that for line 11 . The fluctuations in the outermost region are not as severe as for line 11 . As earlier, the linear and polynomial fits are in close agreement. The values of slope and intercept for the linear fit are -0.024 and 5.71 respectively.
Table 2: Summary of the relative flux density along all 12 lines: The first two columns list the lines (L) and the total number $(\mathrm{N})$ of clicks (i.e., pixels) along each line. The third column gives the total relative flux density $\left(\Sigma \mathrm{F}_{v}\right)$. The fourth column represents the value of relative flux density per pixel $\left(\mathrm{F}_{\mathrm{vp}}\right)$. The last two columns give the background $\left(\mathrm{S}_{\mathrm{BP}}\right)$ and homogeneous background $\left(\mathrm{SH}_{\mathrm{BP}}\right)$ corrected flux per pixel.

\begin{tabular}{|c|c|c|c|c|c|}
\hline $\mathbf{L}$ & $\mathbf{N}$ & $\boldsymbol{\Sigma F}_{\boldsymbol{v}}$ & $\mathbf{F}_{\mathbf{v P}}$ & $\mathbf{S}_{\mathbf{B P}}$ & $\mathbf{S}_{\mathbf{H B P}}$ \\
\hline 1 & 60 & 9441.74 & 157.36 & 136.35 & 85.87 \\
\hline 2 & 58 & 8515.66 & 146.82 & 125.81 & 75.33 \\
\hline 3 & 65 & 9724.00 & 149.60 & 128.59 & 78.11 \\
\hline 4 & 57 & 7876.10 & 138.18 & 117.17 & 66.69 \\
\hline 5 & 68 & 10338.24 & 152.03 & 131.02 & 80.54 \\
\hline 6 & 63 & 9335.56 & 148.18 & 127.17 & 76.69 \\
\hline 7 & 63 & 9532.86 & 151.32 & 130.31 & 79.83 \\
\hline 8 & 69 & 10747.89 & 155.77 & 134.76 & 84.28 \\
\hline 9 & 69 & 10718.55 & 155.34 & 134.33 & 83.85 \\
\hline 10 & 56 & 8529.69 & 152.32 & 131.31 & 80.83 \\
\hline 11 & 73 & 11372.52 & 155.79 & 134.78 & 84.30 \\
\hline 12 & 71 & 10188.34 & 143.5 & 122.49 & 72.01 \\
\hline
\end{tabular}

Table 2 summaries the total relative flux density and the relative flux density per pixel. To correct for the observed values of relative flux density, we obtained two parameters. The first parameter is called background which is the average value of the minimum relative flux density per pixel in the field of view while the other is called homogeneous background which is the average value of relative flux density per pixel in the region just outside the outermost contour ("Background and Homogeneous Background"). It should be noted that the value of relative flux density can be converted into MJy/ str when multiplied by a factor $5.1 \times 10^{-9}$. This unit is astrophysical equivalent to the unit $\mathrm{J} \mathrm{m}^{-2}\left(1 \mathrm{MJy} / \mathrm{str}=9 \times 10^{-20}\right.$ $\left.\mathrm{J} \mathrm{m}^{-2}\right)^{6}$. We can see in Table 2 that the value of relative flux density per pixel is maximum for line 1 and minimum for line 4 .
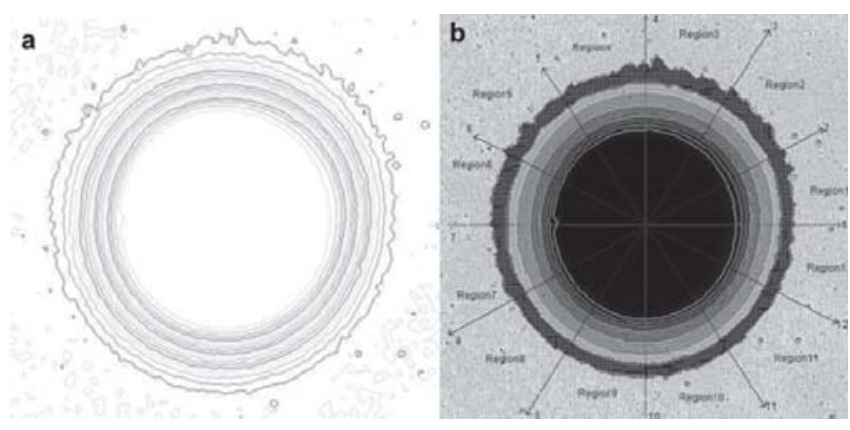

Figure 5: (a) These are contours of iso-potential (in our case relative flux density) surfaces obtained by processing the image of planet Jupiter in the software ALADIN2.5. It should be noted that Jupiter is not in the picture. We can see that the inner contours show mild fluctuations while the outermost two contours show severe fluctuations. (b) Variation of relative flux density in outer region. There are twelve lines passing through the center of the planet Jupiter such that the angle between any two consecutive lines is almost $30^{\circ}$. The region between the outermost two contours is divided into twelve regions by the twelve lines.

\section{DISCUSSION}

In the second quadrant, the value of average relative flux density is found to be minimum. This indicates that the 
absorption of sunlight is prominent in this quadrant. We know that the planet Jupiter radiates twice the amount of heat that it receives from the sun. This heat energy is the remnant of the energy released due to the gravitational contraction during the formation of the planet, On the other hand, the emission of the less amount of energy from the second quadrant is probably due to the materials present in that section as well as due to on going inhomogeneous gravitational contraction ${ }^{7}$. In the third quadrant, the value of average relative flux density is maximum. The first quadrant shows marginally similar value as found in the second quadrant. The difference between the maximum and minimum is $4.25 \%$. This difference cannot be neglected even though it is so small.

As we already discussed that the relative flux density is not identical in the four quadrants. Probably the absorption and reaction take place at the surfaces; therefore it can be suspected that the difference in the values of the relative flux density in the four quadrants is due to the outer atmosphere of the planet Jupiter. So we have decided to study the flux density in the outermost two contours as shown in Fig. 5.

\section{CONCLUSION}

We observed the planet Jupiter from the National Observatory located at Nagarkot, Kathmandu during the period from November, 2008 to January, 2010. The FITS format image of the planet is captured by Meade 1600 LX200GPS SchmidtCassegrain telescope using the software AUTOSTAR SUITE3.08 and the image is processed in the software ALADIN2.5. The values of relative flux density of 500x500 (total 2,50,000) pixels are used for the study. At first, the observed two-dimensional image of the planet is divided into four quadrants. Each quadrant is subdivided into three sections. In this way, we studied the variation of relative ux density along 12 directions. The relative flux density per pixel is found to lie in the range 138.17 to 157.36 . The maximum value of relative flux density per pixel is found along the eastern direction and the minimum value is found along the northern direction. Thus the absorption feature is found to be prominent along the northern direction. The emission of less amount of flux from the second quadrant is due to the absorption by the materials in this section. In addition, the role of the inhomogeneous absorption by the on-going gravitational contraction in the planet cannot be identified. Due to the inhomogeneous absorption features; we studied the outer contours of all sections in details. The values of total amount of flux per pixel in the outer layers of the first, second, third and fourth quadrants are found to be 81.79 , 82.37, 82.44 and 82.49 respectively. Surprisingly, in the first quadrant, this value is found to be minimum. This suggests that the outer part of the planet exhibits a feature opposite to that of the inner part. The outer part is found to be selective whereas the inner part is found to be emissive.

We conclude our result as follows:

(1) The inner part of the planet Jupiter showed homogeneous emission.

(2) The outer part of the planet Jupiter showed inhomogeneous emission. Selective absorption is noticed in particular directions.

(3) The values of relative flux density per pixel are found to be maximum along the eastern direction (line 1).

(4) The minimum value of relative flux density per pixel is found along the northern direction (line 4).

We intend to extend this work by taking several continuous observations in such a way that the atmosphere of the other part of the planet Jupiter can be taken. These observations can lead us to model the outer atmosphere of the planet Jupiter.

\section{ACKNOWLEDGEMENTS}

We acknowledge B.P. Koirala Memorial Planetarium, Observatory and Science Museum Development Board, Ministry of S\&T, Government of Nepal for allotting several nights for the observation. We are highly indebted to Prof. U. Khanal for his constant help and timely suggestion. One of the authors (NRG) acknowledges the board for providing the scholarship for the masters' thesis.

\section{REFERENCES}

1. Karttunen H., Kroger P., Oja H., Poutanen M., Donner K.J. 2007. Fundamental Astronomy Fifth Edition. Springer-Verlag.

2. Encrenaz Th. 1999, Journal Astronomy Astrophysics. Rev. 9: 171.

3. Atreya S. K. 1986, Atmospheres and ionospheres of the outer planets and their satellites, Springer-Verlag.

4. Linfoot E. H., Bradford W. R., Burch C. R., Wayman P. A., Fellgett P. B. 1956. Colloquium on Schmidt optics, The Observatory, Vol.76: 170.

5. Operating Manual 2003: 8", 10", 12", 14", 16" LX200GPS Schmidt-Cassegrain Telescopes, Meade Instruments Corporation.

6. Aumann H. H., Gilespie C. M., Low F. J. 1969. Astrophysical Journal. 57: L69.

7. Belton M. J. S., Ingersoll A. P., Greeley R., McEwen A. S., Klaasen K. P. 1996. Science. 274: 377.

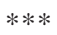

